\title{
Reconfigurable 1.5D Source Arrays for Improved Elevational Focussing in All-Optical Ultrasound Imaging
}

\author{
Erwin J Alles*,†, Sacha Noimark*,†, ${ }^{*}$, Efthymia Papaevangelou ${ }^{\S}$, \\ Edward Z Zhang*, Paul C Beard ${ }^{*} \dagger$ and Adrien E Desjardins ${ }^{*}, \dagger$ \\ *Department of Medical Physics \& Biomedical Engineering, University College London, London, United Kingdom \\ ${ }^{\dagger}$ Wellcome / EPSRC Centre for Surgical and Interventional Sciences, University College London, London, United Kingdom \\ ${ }_{\ddagger}^{\ddagger}$ Materials Chemistry Research Centre, Department of Chemistry, University College London, London, United Kingdom \\ $\S$ School of Immunology and Microbial Sciences, Guy’s Hospital, King’s College London, London, United Kingdom \\ E-mail: e.alles@ucl.ac.uk
}

\begin{abstract}
Recently, an all-optical ultrasound imaging system was presented that was capable of real-time, video-rate 2D pulse-echo imaging. This was achieved through the use of rapid scanning optics and a centimetre-scale optical ultrasound generator, which allowed for the synthesis of 1D source arrays of arbitrary geometry. Back-scattered ultrasound was recorded using a highly sensitive fibre-optic ultrasound detector. Here, a modification to this system is presented that enabled the synthesis of arbitrary 2D source array geometries. This extension allowed for the implementation of 1.5D arrays, which we demonstrated to yield improved elevational resolution $(1.1 \mathrm{~mm}$ for $1 \mathrm{D}$ arrays versus $0.9 \mathrm{~mm}$ for $1.5 \mathrm{D}$ arrays) at the expense of a decrease $(-3.4 \mathrm{~dB})$ in signal-to-clutter level. The axial $(115 \mu \mathrm{m})$ and lateral $(320 \mu \mathrm{m})$ resolutions, as well as the acquisition time, remained unchanged, as was shown using phantoms and $e x$ vivo tissue samples. In addition, the 1.5D source array geometry enabled 3D out-of-plane tracking $(0.7 \mathrm{~mm}$ resolution across a range of $\pm 1.5 \mathrm{~mm}$ ) while performing high-quality 2D imaging. The presented ability to synthesise arbitrary, two-dimensional source array geometries will greatly improve and facilitate ultrasound imaging and guidance of interventional procedures.
\end{abstract}

Index Terms-All-optical ultrasound, reconfigurable array, 1.5D array, elevational resolution, out-of-plane tracking

\section{INTRODUCTION}

All-optical ultrasound is an emerging imaging modality that exhibits great miniaturisation potential, high pressures, wide bandwidths, and resilience to electromagnetic interference. With this modality, ultrasound is generated within an optically absorbing structure via the photoacoustic effect [1], and backscattered pulse-echo signals are recorded using optically resonant structures [2]-[7]. To date, ex vivo biomedical imaging has been performed by mechanically scanning single-element fibre-optic imaging probes across an imaging aperture [2][5], [8]-[12], and in vivo M-mode imaging has been demonstrated [13].

This work was supported by an ERC Starting Grant [310970 MOPHIM], an ERC Advanced Grant [741149], the EU project FAMOS [FP7 ICT, Contract 317744], the Innovative Engineering for Health award by the Wellcome Trust [WT101957] and the EPSRC [NS/A000027/1], the EPSRC Healthcare Technologies Challenge Award [EP/N021177/1], and the Ramsay Memorial Trust. a)

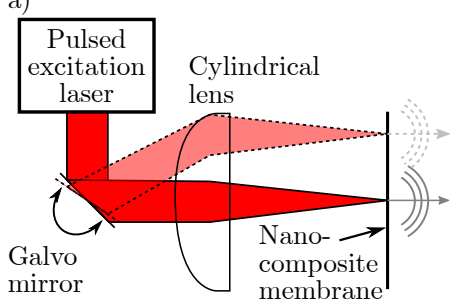

b)

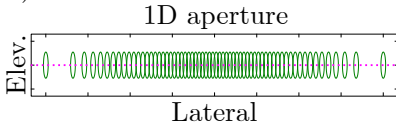

c)

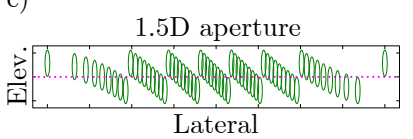

Fig. 1. Imaging paradigm and geometries. (a) Schematic of the all-optical ultrasound imaging paradigm. Eccentrically focussed, pulsed excitation light was delivered to a nanocomposite optical ultrasound generating membrane, where it was converted into ultrasound via the photoacoustic effect. Using a set of galvo mirrors, the resulting optical ultrasound source was scanned across an aperture to synthesise an imaging array. Pulse-echo signals were recorded using a single fibre-optic ultrasound detector (not shown). (b) Schematic of the 1D source aperture geometry used in this work, where a number of eccentric optical ultrasound sources were distributed along a single line. The lateral coordinates of the sources were given by a trigonometric asin distribution. Horizontal ticks: $2 \mathrm{~mm}$ intervals; vertical ticks: $1 \mathrm{~mm}$. To facilitate interpretation, only 64 sources were displayed. (c) Schematic of the $1.5 \mathrm{D}$ source aperture geometry used in this work, where the optical ultrasound sources were distributed along nine parallel lines separated elevationally by $0.25 \mathrm{~mm}$.

Recently, a bench-top all-optical ultrasound imaging system was presented that employed scanning optics to rapidly scan an optical ultrasound source across the surface of an optical ultrasound generator [14]. This system achieved sustained real-time, video-rate imaging (frame rate: up to $20 \mathrm{~Hz}$ ), at lateral and axial resolutions of approximately $100 \mu \mathrm{m}$, an imaging depth of $15 \mathrm{~mm}$, and a dynamic range of $35 \mathrm{~dB}$. In this imaging system, focussed pulsed excitation light was delivered to a large (centimetre-scale) optically absorbing generator membrane. Within the membrane, photoacoustic ultrasound generation occurred only within sharply delineated areas coinciding with the illuminated region, and negligible mechanical cross-talk levels were observed. Consequently, contrary to electronic ultrasound transducers, the resulting optical ultrasound source did not require mechanical isolation such as typically implemented in piezoelectric ultrasound 

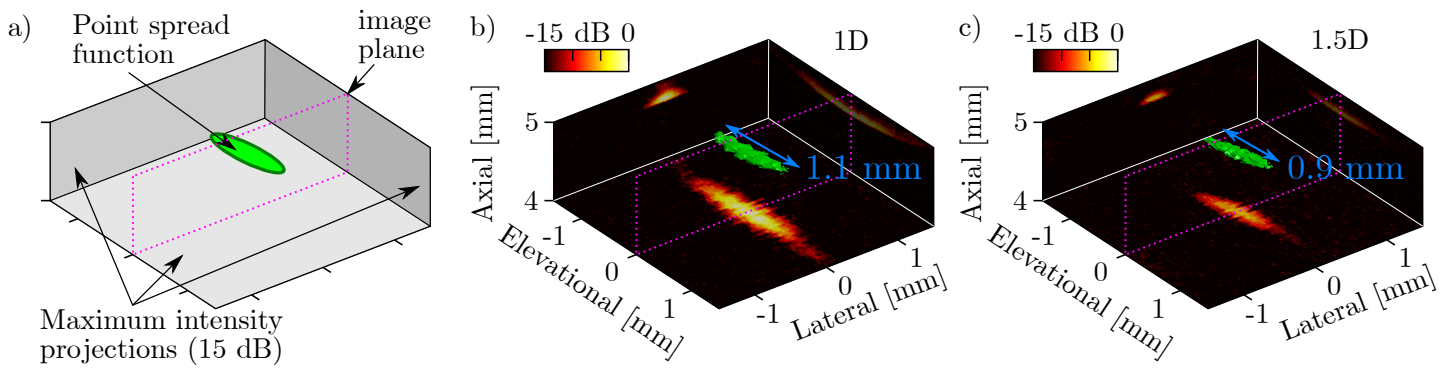

Fig. 2. 3D point-spread functions (PSFs) obtained using 1D and 1.5D source array geometries. (a) Schematic of the information displayed in the point-spread function measurements. A volumetric render of the full-width-at-half-maximum of the PSF is shown in green, whereas the maximum intensity projections (MIPs) along the three axes are shown in the three indicated panels. The 2D image plane of the all-optical ultrasound imaging array is indicated by the purple dashed lines. (b) 3D PSF measurement using a 1D array. The elevational extent of the PSF is indicated in blue. (c) 3D PSF measurement using a $1.5 \mathrm{D}$ array. All of the MIPs are shown on the same logarithmic scale using a $15 \mathrm{~dB}$ dynamic range.

imaging arrays through dicing or electrode patterning. Thus, a large, monolithic optical ultrasound generating canvas was obtained across which an optical ultrasound source could be scanned in arbitrary patterns. As a result, arbitrary source array geometries could be synthesised, such as aperiodic arrays featuring non-uniform source densities and spatially overlapping sources, which allowed for novel image optimisation strategies that increased the image quality and frame rate [14], [15].

In this paper, a modification to this bench-top all-optical ultrasound imaging system is presented where a set of two orthogonal galvo mirrors is used to extend the ability to arbitrarily position an optical ultrasound source to $2 \mathrm{D}$, thus allowing for the synthesis of 2D source arrays of arbitrary geometry. Here, this increased flexibility is used to generate $1.5 \mathrm{D}$ arrays (i.e., 2D arrays of narrow elevational, or outof-plane, extent) to enhance the 2D imaging performance of the system. We demonstrate how such arrays can yield an improved elevational image resolution, and in addition can be used to combine high-quality $2 \mathrm{D}$ imaging with $3 \mathrm{D}$ out-ofplane object tracking.

\section{Methods}

\section{A. Optical ultrasound generation}

As optical ultrasound generator, a thin membrane (thickness: $50 \mu \mathrm{m})$ comprising a nanocomposite of multi-walled carbonnanotubes (MWCNT) and poly-dimethylsiloxane (PDMS) was used, which was previously demonstrated to efficiently transduce light into ultrasound [12], [16]. This membrane was suspended in free-space to avoid spurious reflection artefacts originating from within a rigid backing. Using pulsed laser light (wavelength: $1064 \mathrm{~nm}$, pulse energy: $76 \mu \mathrm{J}$, pulse duration: $5 \mathrm{~ns}$, repetition rate: $3 \mathrm{kHz}$, beam diameter: $1.0 \mathrm{~mm}$; FQS400-1-Y-1064, Elforlight, U.K.), pressures of $0.98 \pm 0.06 \mathrm{MPa}$ were detected at a distance of $2.7 \mathrm{~mm}$, at a bandwidth of $27 \pm 2.2 \mathrm{MHz}$, for optical ultrasound sources positioned arbitrarily across the imaging aperture. Through the use of a cylindrical lens (focal length: $50 \mathrm{~mm}$; LJ1695RMC, Thorlabs, Germany), the excitation light was delivered to an eccentric focal area, resulting in eccentric optical ultrasound sources measuring $224 \pm 53 \mu \mathrm{m}$ (lateral) by $1.10 \mathrm{~mm} \pm 82 \mu \mathrm{m}$ (elevational). This eccentricity improved the directivity of the optical ultrasound sources: the large elevational extent confined the acoustical energy to the imaging plane, thereby maximising the signal-to-noise ratio (SNR) and penetration depth, while the narrow lateral dimensions ensured highresolution $2 \mathrm{D}$ imaging.

\section{B. Imaging aperture synthesis}

Using a set of orthogonal galvo mirrors (GVSM002, Thorlabs, Germany), the excitation light was delivered to arbitrary locations across an imaging aperture measuring $16 \mathrm{~mm} \times 16 \mathrm{~mm}$. By delivering subsequent excitation light pulses to different locations a source aperture could be synthesised, where the optical ultrasound sources fired consecutively in rapid succession (up to 1800 A-scans per second; limited by the galvo mirror inertia). In this work, two geometries were considered: a 1D geometry where a number of sources were distributed along a linear aperture, and a $1.5 \mathrm{D}$ geometry where the same sources were distributed over nine parallel lines separated elevationally by $0.25 \mathrm{~mm}$, thus spanning a narrow elevational aperture of $2 \mathrm{~mm}$. Different numbers of lines or separation distances resulted in higher image artefact levels, smaller improvements in elevational resolution, or both (data not shown).

The lateral coordinate of the $i$-th source, $x_{i}$, was given by the asin distribution,

$$
x_{i}=\frac{2 d}{\pi} \operatorname{asin}\left(2 \frac{i-1}{N-1}-1\right),
$$

where $d=13 \mathrm{~mm}$ is the lateral width of the imaging aperture, and $N$ is the number of optical ultrasound sources. This asin pattern was previously shown to yield a superior 2D image quality compared to a spatially periodic source distribution such as used in conventional electronic ultrasound imaging probes [14]. Here this asin pattern was confirmed to yield higher signal-to-clutter ratios (SCRs) for both 1D and 1.5D source array geometries (data not shown). The imaging paradigm and the 1D and 1.5D array geometries are shown schematically in Fig. 1.

\section{Data acquisition and image reconstruction}

Pulse-echo data were recorded for each optical ultrasound source location using a single, stationary fibre-optic ultra- 
sound detector comprising a Fabry-Pérot cavity [17] (noiseequivalent pressure: $40 \mathrm{~Pa}$, bandwidth: up to $80 \mathrm{MHz}$, omnidirectional response), which was positioned in-plane, laterally centered, and axially offset by $1.0 \mathrm{~mm}$ from the optical ultrasound source aperture. Pressure variations due to impinging ultrasound waves resulted in changes in reflectivity of this cavity [18], which were detected using a photodiode (DET01CFC, Thorlabs, Germany), amplified (+30 dB; DHPVA-200, Femto, Germany), and digitised (125 MSa/s, 14-bit; M4i.4420-x8, Spectrum, Germany) without signal averaging. After acquiring pulse-echo A-scans for all optical ultrasound source locations, power-law time-gain compensation was applied as previously described [14] (where the exponent was empirically determined), and data were reconstructed into 2D images using the Delay-and-Sum algorithm [19].

\section{Point-spread function measurements and object tracking}

The tip of a pushpin (approximately spherical; diameter: $25 \mu \mathrm{m})$ was used to approximate a point scatterer. This pushpin was laterally centered, and scanned elevationally using a motorised translation stage (step size: $50 \mu \mathrm{m}$, range: \pm 1.5 mm; MTS50/M-Z8 + TDC001, Thorlabs, Germany). 2D images were acquired (using 200 optical ultrasound sources) for every pushpin position, and the resulting 61 images were stacked to obtain the 3D point-spread function (PSF). This experiment was performed using both a $1 \mathrm{D}$ and a $1.5 \mathrm{D}$ source array geometry. In addition, the data obtained with the $1.5 \mathrm{D}$ source array geometry were reconstructed into 3D images to investigate the potential for $3 \mathrm{D}$, out-of-plane object tracking that could facilitate needle placement and biopsy guidance.

\section{E. Tissue imaging}

To demonstrate the improved elevational focussing achieved using the 1.5D source array geometry, a 3D image of an excised mouse kidney was acquired ex vivo. This kidney was bonded using cyanoacrylate to cling film $(150 \mu \mathrm{m}$ thick polyvinyl chloride) stretched around an empty frame, placed a distance of $3.5 \mathrm{~mm}$ away from the imaging aperture, and scanned elevationally using a motorised stage (step size: $50 \mu \mathrm{m}$, range: $10 \mathrm{~mm}$ ). 2D images acquired at each elevational position were stacked to obtain a 3D image, from which axial slices were extracted. For this experiment, 1000 optical ultrasound sources were used.

\section{RESULTS}

Measurements of the 3D PSFs revealed that the elevational extent of the PSF was reduced from $1.1 \mathrm{~mm}$ (for a 1D array) to $0.9 \mathrm{~mm}$ when a $1.5 \mathrm{D}$ source array geometry was used (Fig. 2), while the lateral and axial resolutions (320 $\mu \mathrm{m}$ and $115 \mu \mathrm{m}$, respectively) remained unchanged. However, the image intensity decreased by $3.4 \mathrm{~dB}$ due to the increased ultrasound propagation distances and sub-optimal directivity of the (elevationally collimated) optical ultrasound sources.

Similar observations were made in 2D images of a point scatterer obtained with both a 1D and 1.5D array (Fig. 3(a)). Images obtained with the point scatterer located directly within
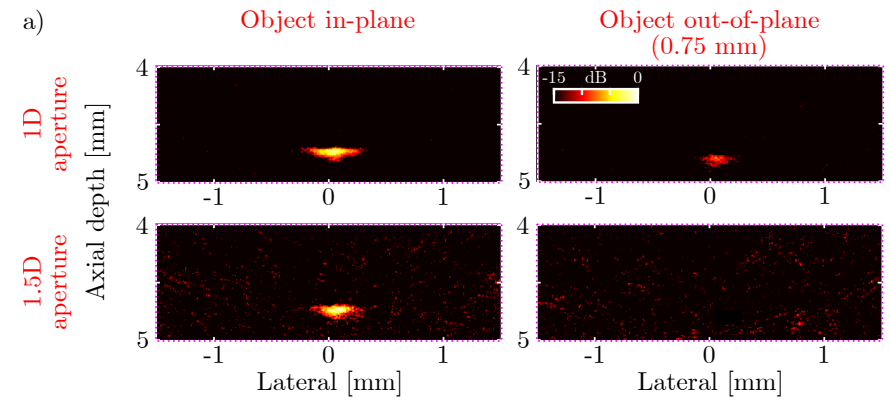

b)

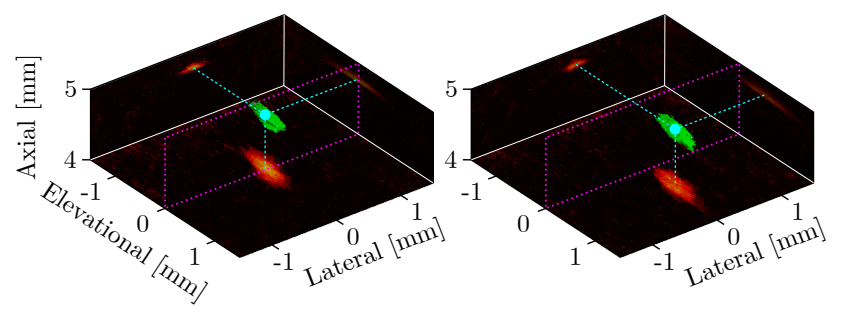

Fig. 3. 2D imaging performance and out-of-plane tracking. (a) $2 \mathrm{D}$ images of a point scatterer placed either directly within (left column) or $0.75 \mathrm{~mm}$ away from the image plane (right column), acquired with either a 1D (top row) or $1.5 \mathrm{D}$ source array geometry (bottom row). (b) $3 \mathrm{D}$ images of a point scatterer placed either directly within (left) or $0.75 \mathrm{~mm}$ away from the image plane (right), acquired using a 1.5D source array geometry. The dotted cyan lines indicate the position of the maximum image intensity. All 2D and 3D images are displayed using the same logarithmic scale at a $15 \mathrm{~dB}$ dynamic range.

the image plane confirmed the lateral and axial resolution were retained, and that the image obtained using the $1.5 \mathrm{D}$ array exhibited a lower SCR. When the point scatterer was positioned $0.75 \mathrm{~mm}$ away from the image plane, this point scatterer was still visible in the case of a 1D array, but suppressed when a $1.5 \mathrm{D}$ array was used.

In addition, the use of a $1.5 \mathrm{D}$ array enabled out-of-plane localisation of the point scatterer up to a resolution of $0.7 \mathrm{~mm}$, for elevational coordinates ranging between $\pm 1.5 \mathrm{~mm}$ (Fig. 3(b)). Outside this elevational range, the SNR was insufficient to locate the point scatterer.

Furthermore, ex vivo imaging of an excised mouse kidney demonstrated how the quality of $2 \mathrm{D}$ images of biologically relevant tissue could be improved through changes in the source array geometry (Fig. 4). Using a 1.5D array, the exterior boundaries and blood vessels of the kidney were visualised more clearly, at the expense of a decrease in SCR.

\section{DISCUSSION AND CONCLUSION}

Through the use of a large (centimetre-scale) optical ultrasound generator membrane and 2D scanning optics, the alloptical ultrasound imaging system presented in this work was capable of synthesising source arrays of arbitrary, twodimensional geometries. Here, we used this flexibility to generate $1 \mathrm{D}$ and $1.5 \mathrm{D}$ source arrays for 2D imaging, and demonstrated how a $1.5 \mathrm{D}$ array geometry yielded an improved elevational resolution, at the expense of a reduction in SCR. Using a $1.5 \mathrm{D}$ array, the axial and lateral resolutions, as well as the acquisition time, remained unchanged, whilst enabling 3D 

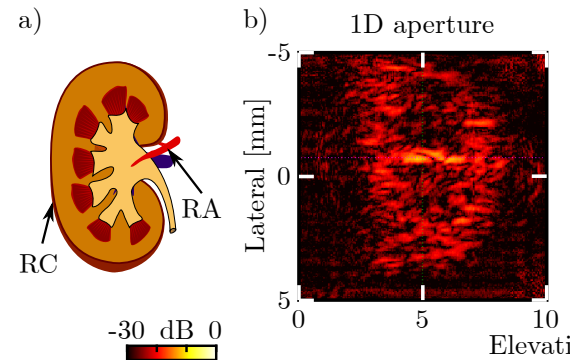

0

Fig. 4. ex vivo imaging of a mouse kidney. (a) Schematic of the anatomy of the mouse kidney. (b) Coronal all-optical ultrasound image through the centre of the mouse kidney obtained using a 1D source array geometry. (c) Coronal all-optical ultrasound image through the centre of the mouse kidney obtained using a 1.5D source array geometry. Both images are displayed on independently normalised logarithmic scales at a dynamic range of $30 \mathrm{~dB}$. RC: renal capsule; RA: renal artery.

out-of-plane object tracking with a system capable of highquality $2 \mathrm{D}$ imaging.

The range and accuracy of the 3D out-of-plane object tracking were limited by the elevational aperture of the $1.5 \mathrm{D}$ array and the directivity of the optical ultrasound sources, which were optimised for 2D imaging. However, the tracking performance can be improved by extending the elevational extent of the 1.5D array, or altering the optical ultrasound source directivity, at the expense of the 2D imaging performance. Similarly, adjusting the source directivity can decrease the reduction in image contrast observed using the $1.5 \mathrm{D}$ array, at the expense of an increased elevational PSF extent.

The ability to synthesise arbitrary two-dimensional source array geometries allows for image quality optimisation paradigms that were hitherto impractical to implement. For example, 1D arrays can be optimised to improve the image quality and frame rate during $2 \mathrm{D}$ imaging, as well as to enable spatially localised image optimisation. 1.5D arrays can be employed to improve the $2 \mathrm{D}$ imaging performance, and to enable 3D out-of-plane object tracking with a system yielding high-quality 2D images. The use of 2D arrays exhibiting significant elevational extent, either densely or sparsely sampled, will enable true 3D imaging [20]. The all-optical ultrasound imaging system presented in this work offers the flexibility to seamlessly switch between any of these imaging modes without changes to its components or layout. Compared to conventional electronic ultrasound imaging probes, where the transducer element positions (and hence mode of operation) are fixed, the flexibility offered by the presented all-optical ultrasound imaging setup will result in increased versatility in operation. Combined with their resilience to electromagnetic interference and MRI compatibility, all-optical ultrasound imaging probes will greatly facilitate and improve ultrasound imaging and guidance of minimally-invasive interventions.

\section{REFERENCES}

[1] PC Beard. Biomedical photoacoustic imaging. Interface Focus, 1(4):602-631, 2011.

[2] Y Hou, J-S Kim, S-W Huang, S Ashkenazi, L Jay Guo, and M O'Donnell. Characterization of a broadband all-optical ultrasound
[2] Y Hou, J-S Kim, S-W Huang, S Ashkenazi, L Jay Guo, and M O'Donnell. Characterization of a broadband all-optical ultrasound transducer-from optical and acoustical properties to imaging. Ultrasonics, Ferroelectrics, and Frequency Control, IEEE Transactions on, 55(8):1867-1877, 2008.

[3] B-Y Hsieh, S-L Chen, T Ling, LJ Guo, and P-C Li. All-optical scanhead for ultrasound and photoacoustic imaging - imaging mode switching by dichroic filtering. Photoacoustics, 2(1):39-46, 2014.

[4] E Biagi, S Cerbai, L Masotti, L Belsito, A Roncaglia, G Masetti, and N Speciale. Fiber optic broadband ultrasonic probe for virtual biopsy: Technological solutions. Journal of Sensors, 2010, 2010.

[5] C Sheaff and S Ashkenazi. Polyimide-etalon all-optical ultrasound transducer for high frequency applications. In Proceedings SPIE BiOS, pages $89434 \mathrm{M}-89434 \mathrm{M}-8$. International Society for Optics and Photonics, 2014.

[6] SM Leinders, WJ Westerveld, J Pozo, PLMJ van Neer, B Snyder, P O'Brien, HP Urbach, N de Jong, and MD Verweij. A sensitive optical micro-machined ultrasound sensor (OMUS) based on a silicon photonic ring resonator on an acoustical membrane. Scientific Reports, 5, 2015.

[7] JA Guggenheim, J Li, TJ Allen, RJ Colchester, S Noimark, OO Ogunlade, IP Parkin, I Papakonstantinou, AE Desjardins, EZ Zhang, and PC Beard. Ultrasensitive plano-concave optical microresonators for ultrasound sensing. Nature Photonics, 11(11):714-719, 2017.

[8] Y Hou, S Ashkenazi, S-W Huang, and M O'Donnell. An integrated optoacoustic transducer combining etalon and black PDMS structures. Ultrasonics, Ferroelectrics, and Frequency Control, IEEE Transactions on, 55(12):2719-2725, 2008.

[9] C Sheaff and S Ashkenazi. An all-optical thin-film high-frequency ultrasound transducer. In Proceedings IEEE IUS, pages 1944-1947. IEEE, 2011.

[10] RJ Colchester, EZ Zhang, CA Mosse, PC Beard, I Papakonstantinou, and AE Desjardins. Broadband miniature optical ultrasound probe for high resolution vascular tissue imaging. Biomedical Optics Express, 6(4):1502-1511, 2015.

[11] EJ Alles, S Noimark, E Zhang, PC Beard, and AE Desjardins. Pencil beam all-optical ultrasound imaging. Biomedical Optics Express, 7(9):3696-3704, 2016.

[12] S Noimark, RJ Colchester, BJ Blackburn, EZ Zhang, EJ Alles, S Ourselin, PC Beard, I Papakonstantinou, IP Parkin, and AE Desjardins. Carbon-nanotube-PDMS composite coatings on optical fibres for alloptical ultrasound imaging. Advanced Functional Materials, 26(35), 2016.

[13] MC Finlay, CA Mosse, RJ Colchester, S Noimark, EZ Zhang, S Ourselin, PC Beard, RJ Schilling, IP Parkin, I Papakonstantinou, and AE Desjardins. Through-needle all-optical ultrasound imaging in vivo: a preclinical swine study. Light: Science \& Applications, 6(12):e17103, 2017.

[14] EJ Alles, S Noimark, E Maneas, EZ Zhang, IP Parkin, PC Beard, and AE Desjardins. Video-rate all-optical ultrasound imaging. Biomedical Optics Express, 9(8):3481-3494, 2018.

[15] EJ Alles, S Noimark, E Maneas, W Xia, EZ Zhang, PC Beard, IP Parkin, and AE Desjardins. Source density apodisation in 2D all-optical ultrasound imaging. In Proceedings IEEE IUS, pages 1-4. IEEE, 2017.

[16] S Noimark, RJ Colchester, RK Poduval, E Maneas, EJ Alles, T Zhao, EZ Zhang, M Ashworth, E Tsolaki, and AH Chester. Polydimethylsiloxane composites for optical ultrasound generation and multimodality imaging. Advanced Functional Materials, 28(9), 2018.

[17] EZ Zhang and PC Beard. Characteristics of optimized fibre-optic ultrasound receivers for minimally invasive photoacoustic detection. In Proceedings SPIE BiOS, pages 932311-932311-9. International Society for Optics and Photonics, 2015.

[18] E Zhang, JG Laufer, and PC Beard. Backward-mode multiwavelength photoacoustic scanner using a planar Fabry-Pérot polymer film ultrasound sensor for high-resolution three-dimensional imaging of biological tissues. Applied Optics, 47(4):561-577, 2008.

[19] M Karaman, P-C Li, and M O'Donnell. Synthetic aperture imaging for small scale systems. Ultrasonics, Ferroelectrics, and Frequency Control, IEEE Transactions on, 42(3):429-442, 1995.

[20] EJ Alles, N Fook Sheung, S Noimark, E Zhang, PC Beard, and AE Desjardins. A reconfigurable all-optical ultrasound transducer array for 3D endoscopic imaging. Scientific Reports, 7, 2017. 